\title{
Vascular Cognitive Impairment
}

By Jonathan Graff-Radford, MD

(1))

CONTINUUM AUDIO INTERVIEW AVAILABLE ONLINE

\begin{abstract}
PURPOSE OF REVIEW: This article provides an overview of vascular cognitive impairment; discusses its epidemiology, subtypes, and associations with other neurodegenerative diseases; and reviews the diagnostic evaluation and management of these disorders.
\end{abstract}

RECENT FINDINGS: Cerebrovascular disease is a common cause of dementia and frequently coexists with neurodegenerative causes. The heterogeneity of mechanisms leading to vascular cognitive impairment makes developing unifying clinical and research criteria difficult. Recognizing the neuroimaging hallmarks of different forms of vascular cognitive impairment can allow for individualized treatment and management. In individuals with mild vascular cognitive impairment, aerobic exercise appears to be a promising treatment but requires further investigation.

SUMMARY: Vascular cognitive impairment can be caused by several mechanisms. While treating vascular risk factors is rational to prevent worsening of cognitive impairment, well-designed studies are needed to demonstrate efficacy.

\section{INTRODUCTION}

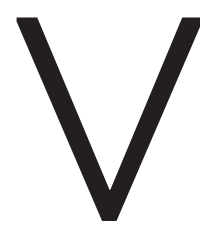

ascular cognitive impairment encompasses a range of disorders in which vascular factors cause or contribute to cognitive decline. Our understanding of vascular cognitive impairment has evolved over time. Traditionally, vascular dementia was clinically distinguished from Alzheimer disease dementia by the course of clinical symptoms, history of vascular disease, and focal findings on neurologic examination. Recent evidence suggests vascular brain diseases are a critical part of the expression of Alzheimer disease and other neurodegenerative diseases. Until pathologic data demonstrated that Alzheimer pathology was the most common etiology of dementia, arteriolosclerosis was thought to be the most common cause of dementia, and doctors attributed dementia to "hardening of the arteries." Later, vascular dementia became synonymous with multi-infarct dementia, which was coined by Hachinski and colleagues, ${ }^{1}$ who emphasized focal neurologic signs and symptoms and "stepwise" cognitive decline.

As neuroimaging improved and large pathologic studies were published, a broader range of vascular diseases resulting in cognitive impairment became appreciated. The term vascular cognitive impairment was introduced to incorporate all types of cognitive impairment related to vascular disease,
CITEAS:

CONTINUUM (MINNEAP MINN) 2019;25(1, DEMENTIA):147-164.

Address correspondence to Dr Jonathan Graff-Radford, Department of Neurology, Mayo Clinic, 200 First St SW, Rochester, MN 55905, Graff-Radford.Jonathan@ mayo.edu.

RELATIONSHIP DISCLOSURE: Dr. Graff-Radford receives research/grant support from the National Institute on Aging/National Institutes of Health (K76AG057015).

UNLABELED USE OF PRODUCTS/INVESTIGATIONAL USE DISCLOSURE:

Dr Graff-Radford discusses the unlabeled/investigational use of acetylcholinesterase inhibitors and memantine for vascular cognitive impairment.

() 2019 American Academy of Neurology. 
including multi-infarct and other vascular diseases causing dementia, those with mild cognitive impairment not meeting criteria for dementia, and those with mixed vascular and degenerative pathology. ${ }^{2}$

\section{DIAGNOSTIC CRITERIA FOR VASCULAR COGNITIVE IMPAIRMENT}

The heterogeneity of clinical phenotypes and vascular pathophysiology affecting the brain complicates the development of unifying criteria for vascular cognitive impairment. For many years, the National Institute of Neurological Disorders and Stroke and the Association Internationale pour la Recherche et l'Enseignement en Neurosciences (NINDS-AIREN) criteria for vascular dementia were used. ${ }^{3}$ These criteria were shown to be specific but not sensitive, with pathologic confirmation. ${ }^{4}$ Several newer criteria have been published but require further clinical and pathologic validation. ${ }^{5-8}$ These newer criteria include a mild cognitive impairment stage and propose subtypes based on mechanism. Despite recent efforts to recognize that vascular cognitive impairment rarely occurs in isolation, current diagnostic criteria have a bias toward considering the diagnosis of vascular cognitive impairment as occurring as a sole etiologic agent in a particular patient.

\section{CLINICAL PRESENTATION}

No typical clinical presentation exists for vascular cognitive impairment. Patients with large-territory strokes may have a stepwise decline and focal signs (eg, hemiparesis), while those with cerebral small vessel disease may present with an insidious onset of cognitive slowing with gait disturbance and parkinsonism. ${ }^{9}$ Patients with a combination of Alzheimer disease and vascular disease may present with an amnestic syndrome that is clinically indistinguishable from pure Alzheimer disease.

\section{NEUROPSYCHOLOGY}

No single neuropsychological pattern distinguishes vascular cognitive impairment from other etiologies of cognitive impairment on an individual basis $^{10}$; however, patterns emerge when studying groups of patients. Patients with vascular cognitive impairment tend to perform worse on tests of executive function compared to memory function. They also have more difficulty with tasks requiring cognitive speed. ${ }^{11}$

\section{EPIDEMIOLOGY}

The epidemiology of vascular cognitive impairment is difficult to study because of the heterogeneity of presentation and limitations of current diagnostic criteria. In the population-based Rotterdam study, which used the conservative NINDS-AIREN criteria, the incidence of vascular dementia was 0.1 per 1000 person-years in those aged 60 to 64 years. ${ }^{12}$ The incidence increased with age to 7.0 per 1000 person-years in those aged 90 to 94 years, with a higher risk of vascular dementia in men. In an Olmsted County, Minnesota, population-based study of autopsied dementia cases, $13 \%$ had pure vascular dementia and an additional $12 \%$ had significant vascular contribution to the pathology, making vascular disease an important component of at least $25 \%$ of dementia cases. ${ }^{13}$ In a community-based clinical pathologic cohort of dementia participants, $38 \%$ had Alzheimer disease and infarcts, 30\% had pure Alzheimer disease pathology, 12\% had infarcts alone, and $4 \%$ had Alzheimer disease with infarcts and Lewy body 
disease pathology, suggesting a role for vascular disease in up to $54 \%$ of dementia cases. ${ }^{14}$

Much of the confusion about the frequency of vascular disease contributing to cognitive impairment reflects the difference between the community and clinical cohorts that have been studied. While community-based and population-based studies have shown vascular cognitive impairment as a common cause of cognitive impairment, ${ }^{14}$ clinical cohorts generally suggest that vascular cognitive impairment is rare. Referral biases in cohorts from memory clinics appear to systematically underrepresent vascular cognitive impairment, in contrast to population-based studies. For example, a significant difference is seen in the pathology underlying a clinical diagnosis of Alzheimer disease between clinical and community cohorts. Mixed Alzheimer and vascular pathology is more common in the community, and pure Alzheimer disease pathology is more common in clinical/referral cohorts. ${ }^{15}$ This was reflected in an autopsy series of 10 university medical centers specializing in Alzheimer disease that found six cases of pure vascular dementia among 1929 autopsied dementia cases. ${ }^{16}$ With the advent of amyloid positron emission tomography (PET) imaging, it became possible to study the frequency of vascular cognitive impairment in the absence of Alzheimer pathology in vivo. In a study of subcortical vascular dementia cases, two-thirds were negative for amyloid deposition, suggesting a vascular dementia phenotype in the absence of Alzheimer disease that can be appreciated antemortem. ${ }^{17}$

\section{Poststroke Dementia}

Many studies have highlighted the association between stroke and the development of dementia. About $10 \%$ of patients have dementia before their first stroke, $10 \%$ develop dementia shortly after their first stroke, and about $33 \%$ develop dementia after a recurrent stroke. ${ }^{18}$ In a population-based study, the incidence of dementia after stroke was 9 times greater than expected. Importantly, dementia risk remained double the control population even when dementia was not present the first year after stroke. ${ }^{19}$ While cortical infarcts were previously emphasized, emerging evidence suggests subcortical infarcts are important risk factors for cognitive impairment. In a follow-up of the SPS 3 (Secondary Prevention of Small Subcortical Strokes) trial, 47\% of participants had mild cognitive impairment. ${ }^{20}$

The criteria for poststroke dementia emphasize a temporal association between the cognitive impairment and infarction, often suggesting a 6-month window. ${ }^{8}$ While this is helpful in establishing a relationship between the cognitive impairment and vascular disease, numerous studies have highlighted the importance of "silent" infarcts (the presence of at least one infarct detected on MRI without a clinical history of an associated stroke or transient ischemic attack) in the development of dementia. Approximately $20 \%$ of the population older than 65 years of age have silent infarctions that increase the risk of developing dementia. ${ }^{21}$

\section{VASCULAR COGNITIVE IMPAIRMENT SUBTYPES}

O'Brien and colleagues ${ }^{2}$ have proposed the following subtypes of vascular cognitive impairment: multi-infarct dementia, small vessel dementia, strategic infarct dementia, hypoperfusion dementia, hemorrhagic dementia, hereditary vascular dementia, and mixed dementia. Recent pathologic data support
KEY POINTS

Vascular cognitive impairment represents a spectrum of vascular disorders that cause cognitive impairment.

No single neuropsychological pattern distinguishes vascular cognitive impairment from other etiologies of cognitive impairment; however, patients with vascular cognitive impairment tend to perform worse on tests of executive function compared to memory function.

In the community setting, cerebrovascular disease commonly occurs with neurodegenerative diseases.

Both clinical and so-called "silent" strokes are significant risk factors for the development of dementia. 
microinfarcts as an important pathologic substrate of vascular cognitive impairment often occurring with other vascular pathologies.

Neuroimaging has allowed improved understanding of the substrate underlying vascular cognitive impairment. Dividing vascular pathologies detected on brain MRI into broad mechanistic categories is another way to categorize them: small vessel disease associated with hypertensive arteriopathy, small vessel disease related to cerebral amyloid angiopathy, and large vessel/embolic disease. Often individuals with vascular cognitive impairment have more than one of these vascular mechanisms present. This division allows individual patients to be classified and interventions to be tailored to mechanism.

Cerebral Small Vessel Disease Associated With Hypertensive Arteriopathy Prior criteria published for subcortical vascular dementia referred to lacunar strokes and white matter hyperintensity in a subcortical pattern. ${ }^{22}$ Lacunar stroke and white matter hyperintensity are associated with a hypertensive arteriopathy, although other vascular risk factors such as diabetes mellitus and smoking contribute as well. FIGURE 7-1 demonstrates the spectrum of imaging changes with hypertensive arteriopathy.

DeEP CEREBRAL MICROBLeeds. Microbleeds are detected on hemosiderin-sensitive MRI sequences $\left(\mathrm{T} 2{ }^{*}\right.$ gradient recalled echo [GRE] and susceptibility-weighted imaging [SWI]). Deep cerebral microbleeds are thought to be a manifestation of hypertensive vascular disease and are associated with the presence of lacunar stroke ${ }^{23}$ and decline in motor functioning. ${ }^{24}$ They are associated with the

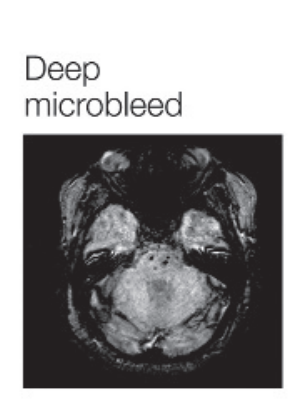

\section{Deep MRI visible perivascular spaces}
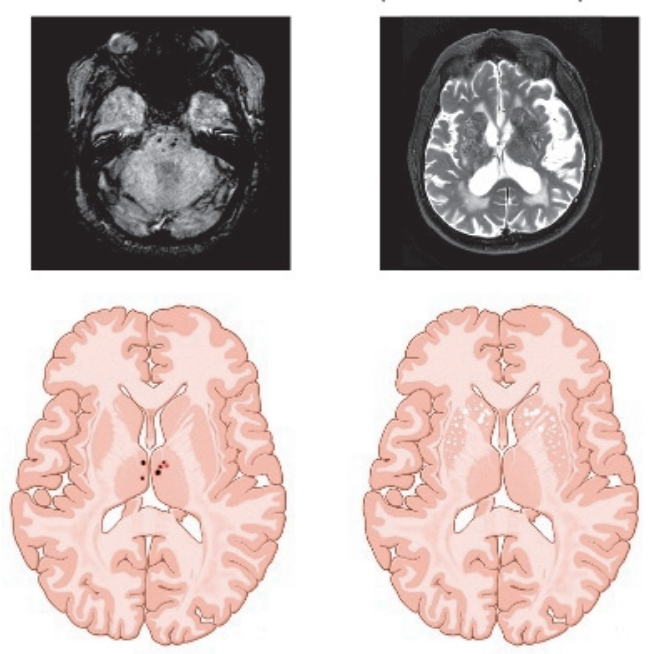
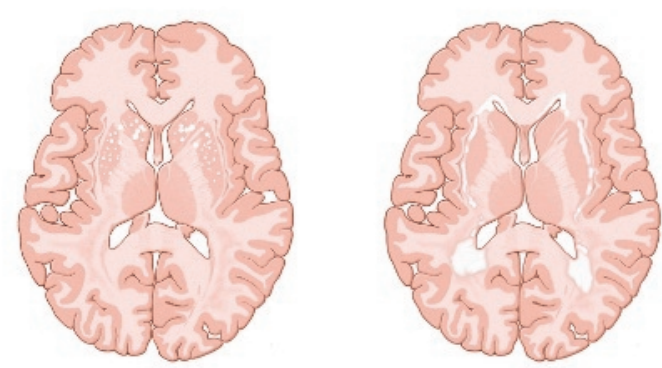

a. Peri-basal ganglia
White matter hyperintensity/ lacunar stroke
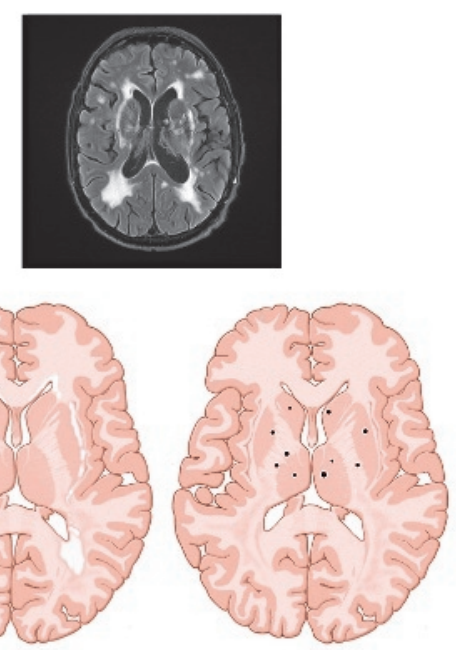

b. Lacunar stroke

\section{Deep}

hemorrhage
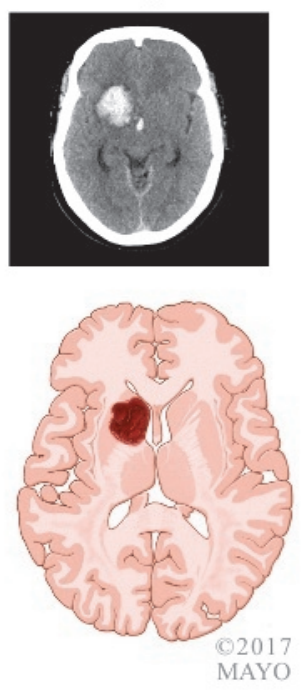

FIGURE 7-1

Spectrum of imaging changes with hypertensive arteriopathy. $\mathrm{MRI}=$ magnetic resonance imaging. 
increased risk of developing a deep (hypertensive) hemorrhage. They are also associated with risk of ischemic stroke. ${ }^{25}$ In patients with Alzheimer dementia, the presence of deep cerebral microbleeds was associated with increased cardiovascular mortality. ${ }^{26}$

BASAL GANGLIA (DEEP) MRI-VISIBLE PERIVASCULAR SPACES. The presence of basal ganglia (deep) enlarged perivascular spaces is associated with deep cerebral microbleeds, hypertension-related intracerebral hemorrhage, and increased white matter T2 hyperintensity burden. ${ }^{27}$ In one study, increased burden of basal ganglia perivascular spaces was associated with a diagnosis of subcortical vascular cognitive impairment and negatively predicted the diagnosis of Alzheimer dementia. ${ }^{28}$

WHITE MATTER HYPERINTENSITIES. White matter T2 hyperintensities are commonly detected on brain MRI and increase with age. The presence of white matter T2 hyperintensities is increasingly recognized as detrimental because of their association with an increased risk of stroke, dementia, ${ }^{29}$ and, particularly, worsening cognitive speed and executive function. Noncognitive correlates include risk of depression ${ }^{30}$ and gait impairment. ${ }^{31}$ Progression of white matter hyperintensity over time is associated with cognitive decline. ${ }^{32}$ While white matter $\mathrm{T} 2$ hyperintensities are often considered vascular in origin because of the association with vascular risk factors such as hypertension, ${ }^{33}$ the location of the hyperintensities is important for understanding the underlying pathophysiology. Hyperintensities in deep regions are more common in individuals with a hypertensive intracerebral hemorrhage than in those with a cerebral amyloid angiopathy-related hemorrhage. ${ }^{34}$ In patients with Alzheimer dementia, parietal hyperintensity may reflect Alzheimer neuropathology rather than a vascular origin. ${ }^{35}$ The cause of white matter $\mathrm{T} 2$ hyperintensity can be heterogeneous, particularly in younger individuals with genetic, inflammatory, or radiation-induced changes. Therefore, the presence of white matter hyperintensity should not be considered as definitive evidence of cerebrovascular disease.

LACUNAR STROKES. While lacunar infarcts commonly occur in the setting of increased white matter T2 hyperintensities, both are independently associated with worse cognition. ${ }^{36}$ The location of lacunar infarct is key. Thalamic and putaminal locations are typically associated with a worse cognitive performance than capsular, caudate, and white matter lacunar strokes. ${ }^{37}$ While the location of lacunar stroke often predicts the cognitive domain involved, overall incident lacunar infarcts predict decline in executive functions and psychomotor speed. ${ }^{38}$ In the Nun Study, participants with Alzheimer pathology were much more likely to develop dementia if they also had lacunar strokes. ${ }^{39}$

\section{Small Vessel Disease Related to Cerebral Amyloid Angiopathy}

Cerebral amyloid angiopathy is caused by the pathologic deposition of amyloid- $\beta$ within the walls of cerebral blood vessels, leading to weakening of blood vessels that increases the risk of hemorrhage. Cerebral amyloid angiopathy often occurs with Alzheimer disease pathology but is also a common pathology of aging and prevalent in community-based autopsy studies. ${ }^{40}$ While cerebral amyloid angiopathy may be a risk factor for hemorrhagic stroke, it is also associated with cognitive impairment even in the absence of clinical hemorrhage. Severe cerebral 
amyloid angiopathy pathology is associated with impaired episodic memory and decreased perceptual speed even after controlling for coexisting Alzheimer disease pathology. ${ }^{40}$ In the Honolulu-Asia Aging Study, patients with Alzheimer disease with coexisting cerebral amyloid angiopathy had worse cognitive performance that those without coexisting cerebral amyloid angiopathy. ${ }^{41} \mathrm{Clinical}$ criteria (the Boston criteria) for the diagnosis of cerebral amyloid angiopathy have been developed and modified to include superficial siderosis. ${ }^{42,43}$

Several neuroimaging biomarkers for the presence of cerebral amyloid angiopathy exist and are summarized in FIGURE 7-2, including lobar microbleeds, lobar intracerebral hemorrhage, cortical superficial siderosis, white matter hyperintensity, convexity subarachnoid hemorrhage, and MRI-visible centrum semiovale perivascular spaces.

LOBAR CEREBRAL MICROBLeEDS. Lobar cerebral microbleeds are an established risk factor for intracerebral hemorrhage. ${ }^{44}$ The presence of multiple lobar cerebral microbleeds is associated with increased risk of dementia and impaired executive function and memory. ${ }^{24}$ In patients with Alzheimer dementia, lobar cerebral microbleeds are associated with an increased risk of stroke-related mortality. ${ }^{26}$

LOBAR INTRACEREBRAL HeMORRHAGE. Among individuals without dementia who develop an intracerebral hemorrhage, the incidence of dementia is approximately $28 \%$ at 4 years, highest among those with imaging features suggestive of underlying cerebral amyloid angiopathy, including a lobar location. ${ }^{45}$

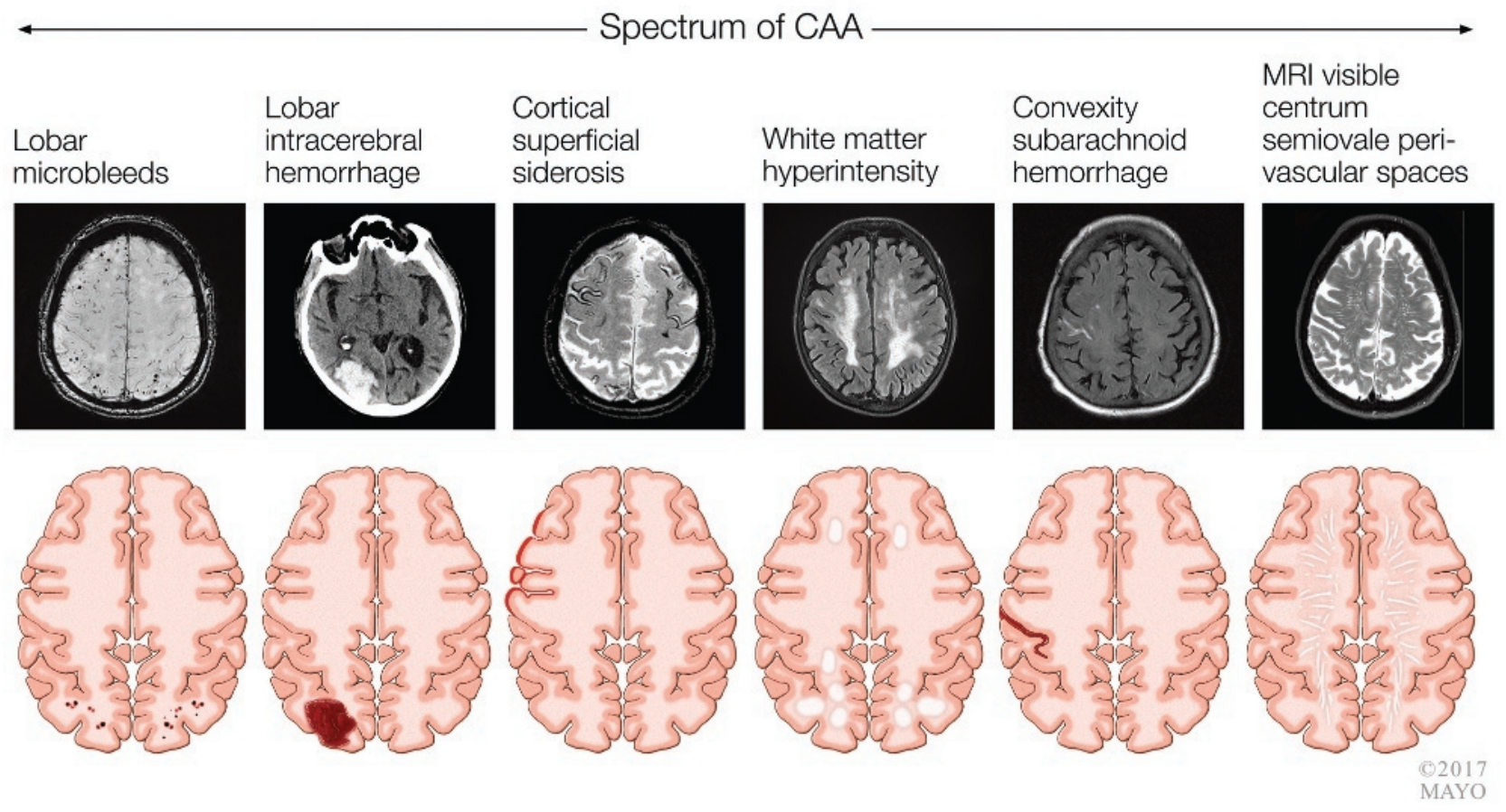

FIGURE 7-2

Spectrum of imaging changes associated with cerebral amyloid angiopathy (CAA). $\mathrm{MRI}=$ magnetic resonance imaging. 
CORTICAL SUPERFICIAL SIDEROSIS. Cortical superficial siderosis refers to curvilinear hemosiderin deposition along superficial layers of the cerebral cortex; it is a predictor of intracerebral hemorrhage risk. ${ }^{46}$

CONVEXITY SUBARACHNOID HEMORRHAGE. LesS commonly, cerebral amyloid angiopathy can present with convexity subarachnoid hemorrhage, often presenting with recurrent transient spreading sensory and motor symptoms.

MRI-VISIBLE CENTRUM SEMIOVALE PERIVASCULAR SPACES. Centrum semiovale enlarged perivascular spaces occur more commonly in cerebral amyloid angiopathy-related intracerebral hemorrhage than in hypertensive intracerebral hemorrhage and are also detected more frequently in Alzheimer dementia than in vascular cognitive impairment. ${ }^{28}$

AMYLOID POSITRON EMISSION TOMOgRAPHY. Amyloid PET has been explored as a biomarker of cerebral amyloid angiopathy and appears to be able to distinguish hemorrhages caused by hypertension from those caused by cerebral amyloid angiopathy. ${ }^{47}$ Although preliminary studies of amyloid PET are promising, further studies with pathologic confirmation are necessary before amyloid PET can be used to reliably identify cerebral amyloid angiopathy clinically.

\section{Strategic Infarction}

Strategic strokes can be either small vessel (lacunar) or large vessel in mechanism. Targeted strokes can present with cognitive deficits, including memory loss. The cause can often be distinguished from neurodegenerative disease by the time course, which is sudden in onset with vascular causes. The NINDS-AIREN criteria identify several brain regions in key cognitive networks associated with the development of strategic cognitive impairment, including angular gyrus, thalamus (CASE 7-1), basal forebrain, posterior cerebral artery (ie, hippocampus) (CASE 7-2), and anterior cerebral artery (CASE 7-3) territories, which can be injured after rupture of an anterior communicating artery aneurysm. ${ }^{3}$ Other regions include the caudate (CASE 7-4) and putamen. While the area of injury may be small, these lesions cause cognitive deficits by disrupting widespread brain networks that can be defined with functional neuroimaging.

\section{Large Vessel Disease}

Two imaging manifestations of large vessel disease are shown in FIGURE 7-6. As reviewed above, strategic infarct dementia can also be a manifestation of large vessel disease. Intracranial atherosclerosis has been associated with dementia, adjusting for the presence of vascular risk factors. ${ }^{51}$ This association is supported by pathologic data. ${ }^{52,53}$ In contrast, extracranial carotid-intima thickness has not consistently been associated with cognitive decline. ${ }^{54}$

MULTI-INFARCT DEMENTIA. Multi-infarct dementia was originally conceptualized by Hachinski and colleagues ${ }^{1}$ as a condition in which multiple cortical infarcts of different size and location led to cognitive impairment. Multi-infarct dementia now represents a small subset of individuals with vascular cognitive impairment. These patients may have an abrupt onset to their symptoms, with a stepwise neurologic decline and focal neurologic findings on examination (CASE 7-5). Imaging reveals multiple cortical strokes. Atrial fibrillation, which can be the underlying cause of multiple cortical strokes, is
KEY POINTS

Neuroimaging biomarkers may allow for identification of different mechanisms leading to small vessel disease. For example, deep cerebral microbleeds are suggestive of hypertensive arteriopathy, and lobar cerebral microbleeds are suggestive of cerebral amyloid angiopathy.

Several strategic brain regions have been associated with the development of dementia after an infarct, including the angular gyrus, thalamus, caudate and putamen, basal forebrain, posterior cerebral artery (ie, hippocampus), and anterior cerebral artery territories. 
associated with cognitive decline even without a history of clinical stroke. The association of atrial fibrillation with cognitive impairment varies by the presence of subclinical "silent" infarctions. ${ }^{55}$

HYPOPERFUSION. Cardiac arrest, carotid occlusion, and hypotension may lead to watershed infarcts (FIGURE 7-6), anoxic brain, or incomplete white matter infarcts, which can result in cognitive impairment.

\title{
Genetic Causes
}

Several genetic disorders are associated with the development of vascular cognitive impairment. Cerebral autosomal dominant arteriopathy with

\section{CASE 7-1}

COMMENT

\begin{abstract}
A 75-year-old man with a history of hypertension presented for a second opinion regarding memory loss. He repeatedly asked his wife "Can you tell me where I am?" In the emergency department, he was initially diagnosed with transient global amnesia, but his symptoms did not improve, so an MRI was ordered, which revealed an ischemic infarct in the anterior nucleus of the thalamus (FIGURE 7-3). After hospital discharge, he had persistent anterograde amnesia and had difficulty with navigation; his wife reported that he got lost in his own house and would go into the closet looking for the bathroom. The stroke was attributed to small vessel disease from his hypertension.
\end{abstract}

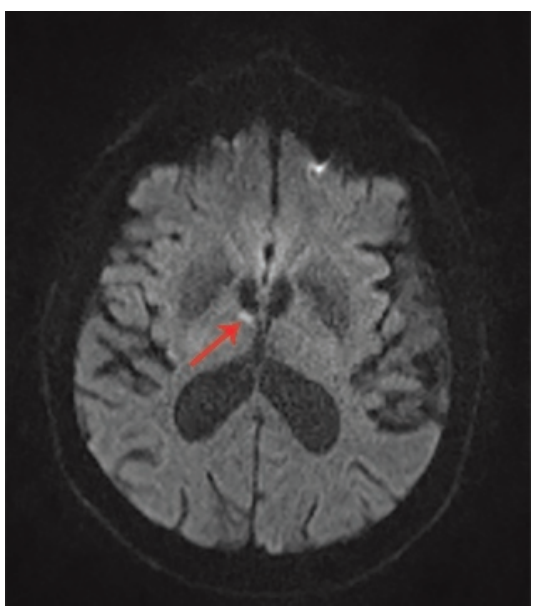

FIGURE 7-3

Imaging of the patient in CASE 7-1. Axial diffusion-weighted MRI shows an acute ischemic infarct in the anterior nucleus of the thalamus (arrow).

The anterior nucleus of the thalamus is an important part of the network for memory, which includes the cingulate gyrus, hippocampus, mammillary body, and orbital and medial frontal lobes. The anterior nucleus of the thalamus receives blood supply from the tuberothalamic artery, which originates from the posterior communicating artery. The clinical phenotype of anterior nucleus infarction is anterograde amnesia with preserved recognition. The amnesia results from interruption of the mammillothalamic tract and its cortical projections. Patients with left anterior nucleus infarction typically have verbal memory difficulty and autobiographical memory impairment, while patients with right anterior nucleus infarction, such as this patient, develop topographic disorientation and visuospatial dysfunction. Patients with anterior thalamic strokes have also been described to develop palipsychism, which is superimposition of temporally unrelated information. ${ }^{48}$ This presents as an interruption of current dialogue with previously discussed unrelated topics. 
subcortical infarcts and leukoencephalopathy (CADASIL) is a genetic cerebral small vessel disease caused by mutations in the $\mathrm{NOTCH}_{3}$ gene that codes for a transmembrane receptor located on vascular smooth muscle cells (CASE 7-6).

This leads to deposition of granular osmophilic material in vascular smooth muscle cells. Cognitive impairment is common, as are migraine headaches and stroke. The pattern of cognitive deficits on neuropsychometric testing includes impaired cognitive speed, executive function, and attention..$^{56}$ MRI reveals significant white matter T2 hyperintensities with ischemic lesions in subcortical regions, including characteristic involvement of the anterior temporal lobe.

Cerebral autosomal recessive arteriopathy with subcortical infarcts and

A 73-year-old woman was brought to the emergency department by her family after she sent them a series of unusual emails. Her family members indicated that she was acting "weird." Her email read "Thank you for getting my mail and for bringing me home. THANK YOU for hauling me home and all my things. Many thanks for coming to get me and hauling my stuff in. I cannot even remember if I thanked you. I do not mean to forget my thanks when you do things that help so much for me. Thank you for bringing me home and hauling my things in. Makes me sad when I forget to say thank you."

In the emergency department, her neurologic examination was notable for a right homonymous hemianopia that resolved by the next morning. She was unable to recall four words 5 minutes after they were given to her. She also had a visual object agnosia; despite normal visual fields and intact reading, she was unable to name objects presented to her in the visual sensory modality, but she correctly named them when presented by auditory or tactile sensory modalities.

MRI revealed an infarction of the left hippocampus as well as the left ventral visual stream (occipitotemporal area) (FIGURE 7-4). When she was seen 6 months later, her memory and visual agnosia had not improved.

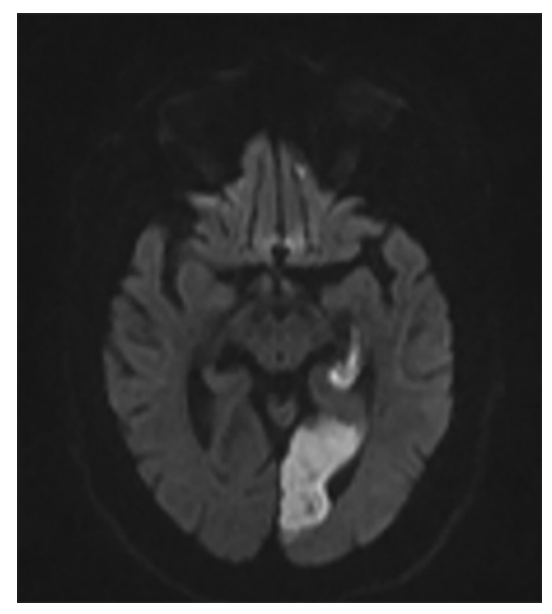

FIGURE 7-4

Imaging of the patient in CASE 7-2. Axial diffusion-weighted MRI shows an acute infarction in the left posterior cerebral artery distribution involving the left hippocampus and the left ventral visual stream (occipitotemporal area), resulting in dementia related to a strategic infarct.

This case illustrates that strategic infarcts can result from large artery 
leukoencephalopathy (CARASIL), another hereditary vascular dementia syndrome, occurs due to mutations in the HTRA1 gene and is associated with alopecia and spondylosis in addition to the cerebral small vessel disease. ${ }^{57}$

Familial cerebral amyloid angiopathy has been described with mutations in the $A P P, I T M 2 B$ (or BRI2), and CST3 genes. ${ }^{58-60}$

\title{
Mixed Dementia
}

Mixed dementia is most often seen as combined cerebrovascular disease and Alzheimer disease. As demonstrated by several community-based pathologic cohorts, mixed vascular and degenerative pathology is perhaps the most prevalent important cause of cognitive impairment. The presence of vascular pathology lowers the threshold for the clinical expression of Alzheimer disease. The presence of infarcts interacts with Alzheimer pathology to increase the likelihood of dementia. ${ }^{61}$ In the Baltimore Longitudinal Study of Aging, the presence of an infarction significantly increased the odds ratio for dementia independently of whether the infarct was symptomatic. ${ }^{62}$ The overlap can also be seen in epidemiologic studies in which Alzheimer disease dementia and vascular cognitive impairment share treatable vascular risk factors, including hypertension, dyslipidemia, and diabetes mellitus. ${ }^{62,63}$

\section{Microinfarcts}

Recent evidence suggests microinfarcts are an important contributor to dementia. In one community-based sample, the population-

attributable risk of dementia for microinfarcts was $33 \% .{ }^{64}$ The pathophysiology of developing microinfarcts has recently been investigated. Cortical microinfarcts are associated with the presence of cerebral amyloid angiopathy pathology, while deep microinfarcts are associated with the presence of arteriosclerosis. ${ }^{65}$ Interestingly, brain atrophy in a watershed pattern is associated with the presence

\section{CASE 7-3}

\begin{abstract}
A 60-year-old man was hospitalized following rupture of an anterior communicating artery aneurysm complicated by vasospasm. After several weeks, he had no focal neurologic deficits, but he had confabulation and amnesia. He had previously worked as a car mechanic, and on morning rounds in the hospital, when asked what he had done the day before, he would describe the type of engine he was "repairing" and where he was going to drop it off. He would incorporate objects present in the room or what he was watching on the television into his confabulations. Head CT demonstrated bilateral anterior cerebral artery infarcts secondary to vasospasm with the associated artifact from his aneurysm treatment.
\end{abstract}

COMMENT

In 1985, Damasio and colleagues ${ }^{49}$ described the syndrome of basal forebrain injury after anterior communicating artery aneurysm rupture or tumor resection. Patients with this syndrome are able to learn separate modal stimuli but cannot integrate the information. They also develop confabulation. Performance in patients with this syndrome is significantly improved by cuing. 
A 60-year-old man presented for evaluation of behavioral changes.

Several months earlier, he developed sudden-onset facial droop, which resolved within 24 hours. MRI revealed a right caudate infarct. Upon returning to work, he received a poor work evaluation, which was uncharacteristic of him. In addition, he shrugged off the criticism, which, his wife noted, was very unusual. He also developed lack of empathy and a sweet tooth, eating ice cream daily for breakfast. Neuropsychological testing showed impairment on the Wisconsin Card Sorting Test. Fludeoxyglucose positron emission tomography (FDG-PET) revealed hypometabolism in areas functionally connected to the caudate (FIGURE $7-5^{50}$ ).
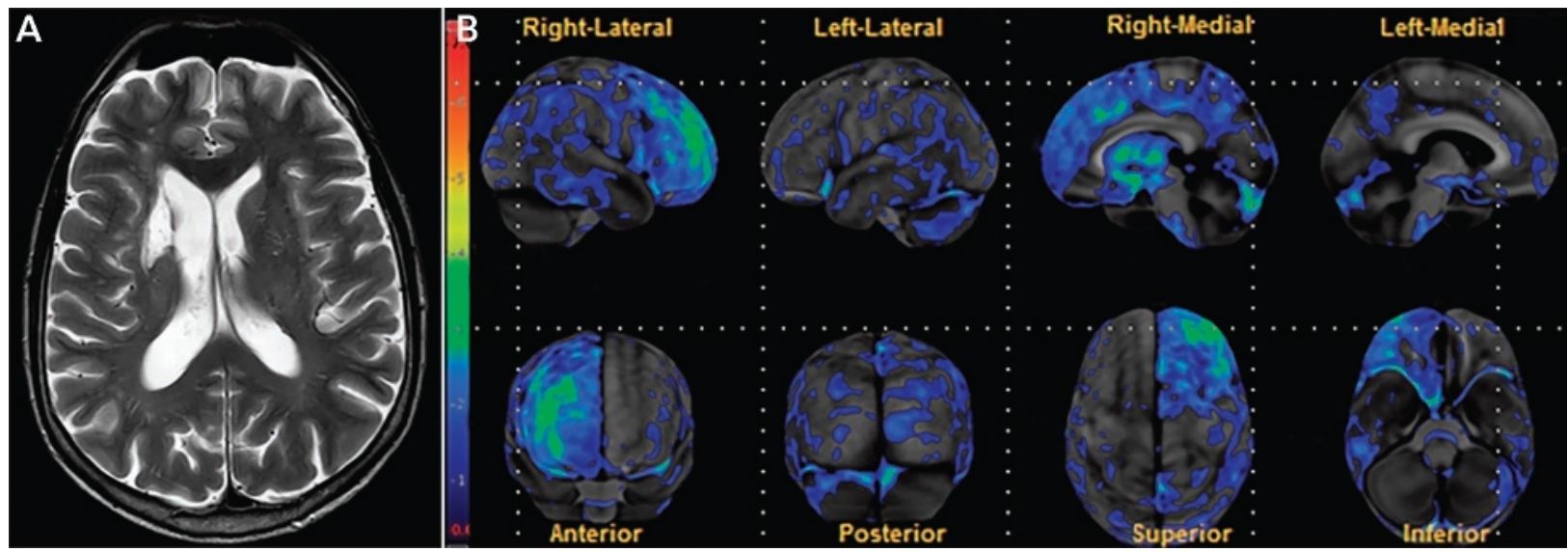

FIGURE 7-5

Imaging of the patient in CASE 7-4. A, Axial T2-weighted MRI shows an infarct involving the right caudate. $B$, Fludeoxyglucose positron emission tomography (FDG-PET) statistical map shows regions of significant hypometabolism relative to age-matched controls.

Hypometabolism is present in areas functionally connected to the caudate, including the right medial prefrontal cortex, dorsolateral prefrontal cortex, and right anterior cingulate cortex. Contralateral cerebellar hypometabolism is also seen.

Reprinted with permission from Graff-Radford J, et al, Neurology. ${ }^{50} \odot 2017$ American Academy of Neurology.

This case demonstrates how a strategic stroke can mimic the symptoms of behavioral variant frontotemporal dementia. In this patient, a single lesion disrupted the connectivity of the caudate to the frontal lobe, resulting in dorsolateral, medial prefrontal, and anterior cingulate cortical dysfunction that manifested as corresponding disinhibition, apathy, loss of empathy, and increased cravings for sweets. 
Multiple cortical strokes
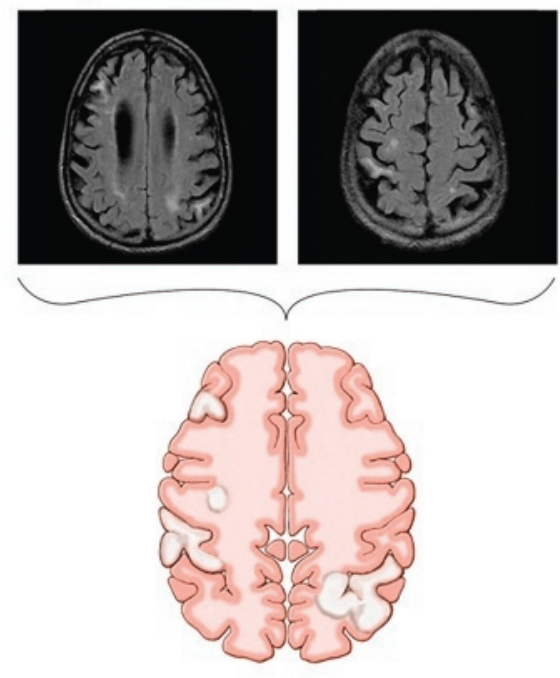

Watershed stroke
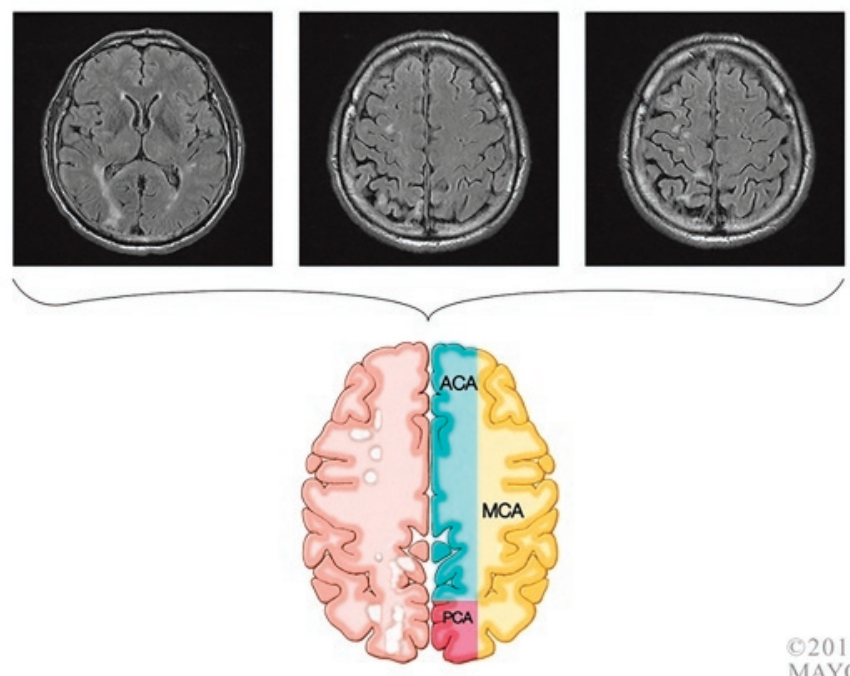

FIGURE 7-6

Embolic disease and large vessel disease. Multiple cortical infarcts (left) due to cardiogenic emboli and watershed infarct (right) related to hypoperfusion in the setting of a significant carotid stenosis.

$\mathrm{ACA}=$ anterior cerebral artery; $\mathrm{MCA}=$ middle cerebral artery; $\mathrm{PCA}=$ posterior cerebral artery .

\section{CASE 7-5}

A 69-year-old right-handed man presented to the behavioral neurology clinic with cognitive slowing, visuospatial difficulty, and trouble multitasking. He indicated that his symptoms started abruptly 2 years earlier, when he developed left-sided weakness and was diagnosed with a stroke.

His neuropsychological examination revealed significantly slowed cognitive speed, impaired cognitive flexibility (ie, perseverations), and higher-order cognitive-set maintenance abilities (ie, executive skills). He scored 11/15 on the delayed recall portion of the Rey Auditory Verbal Learning Test. His neurologic examination was notable for pronator drift on the left and left greater than right hyperreflexia. His MRI revealed multiple areas of cortical infarction. 
A 49-year-old woman presented after developing sudden-onset

left-sided weakness. MRI showed a right internal capsule stroke. She was placed on aspirin and clopidogrel. Four months later, she developed dizziness and confusion. MRI demonstrated a second stroke. She was referred to the cerebrovascular clinic for a second opinion given her two strokes and young age. Her mother had a stroke in her sixties, developed dementia, and died in her seventies. Her maternal uncle also had strokes in his sixties. Her sister had a stroke when she was 53 . The patient had no history of migraine. Her MRI was notable for subcortical ischemic infarcts in addition to white matter T2 hyperintensities with involvement of the anterior temporal lobes and external capsule (FIGURE 7-7). Genetic testing confirmed the pathogenic mutation in the NOTCH3 gene, confirming cerebral autosomal dominant arteriopathy with subcortical infarcts and leukoencephalopathy (CADASIL).
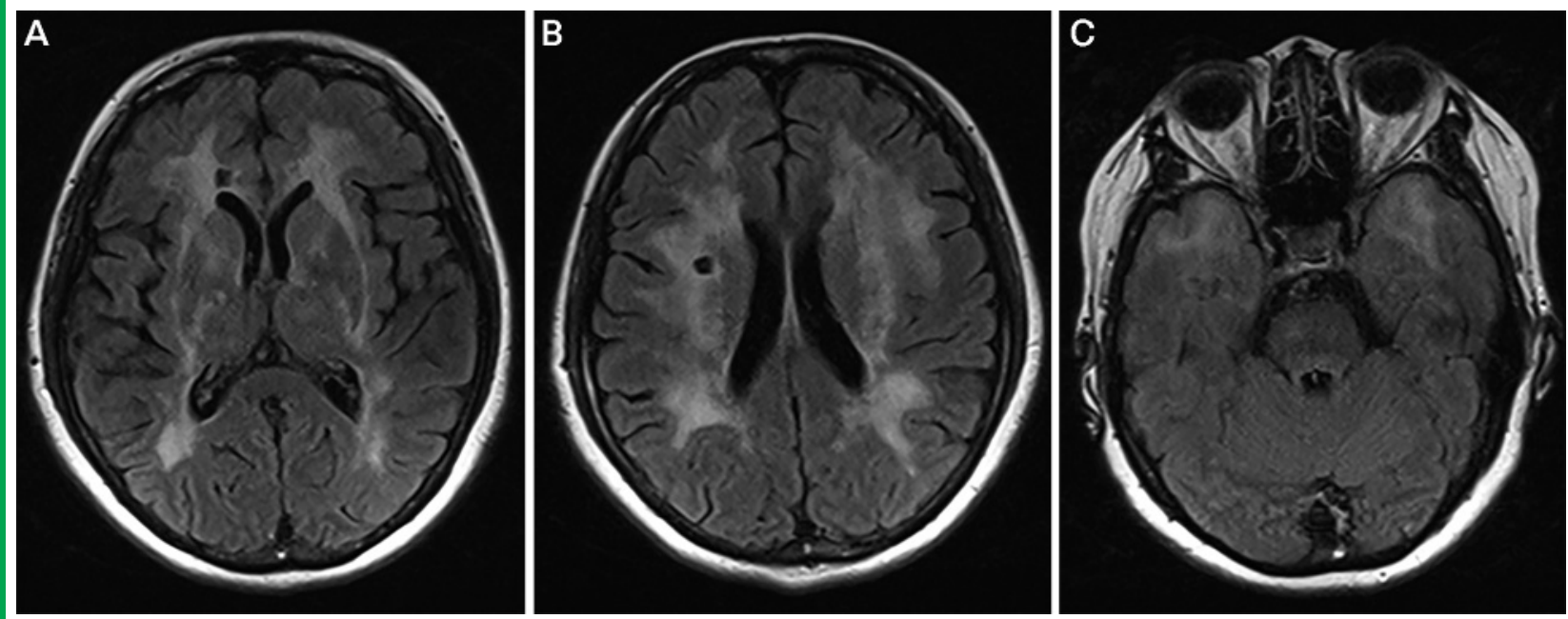

\section{FIGURE 7-7}

Imaging of the patient in CASE 7-6. Axial fluid-attenuated inversion recovery (FLAIR) MRI shows subcortical ischemic infarcts $(A, B)$ in addition to white-matter T2 hyperintensities with notable involvement of the anterior temporal lobes $(C)$ and external capsule $(A)$ characteristic of cerebral autosomal dominant arteriopathy with subcortical infarcts and leukoencephalopathy (CADASIL).

In this patient, CADASIL was suspected because of her young age, typical MRI findings, and family history. CADASIL can also be diagnosed via skin biopsy to examine the walls of the vascular smooth muscle cells by electron microscopy, which can demonstrate irregular deposits of granular osmophilic material. 
of microinfarcts after controlling for Alzheimer pathology. ${ }^{66}$ In addition, subcortical microinfarcts are associated with declining blood pressure over time. ${ }^{67}$

Imaging studies have identified cerebral microinfarcts with 7 T MRI. A subset of these is also visible with $3 \mathrm{~T} \mathrm{MRI} .{ }^{68}$ Since the majority of microinfarcts detected pathologically are approximately 0.2 millimeters in size, these imaging techniques detect a fraction of microinfarct burden.

\section{TREATMENT}

Overall management of vascular cognitive impairment, similar to most other dementias, emphasizes education, counseling, and support of patients and caregivers; home safety evaluation (including medication administration and assessment of driving safety); and advance care planning. While aggressive treatment of vascular risk factors to prevent cognitive decline due to vascular disease seems logical, only a paucity of evidence exists at this time to support such an approach.

\section{Symptomatic Treatment}

The American Heart Association/America Stroke Association have published recommendations for the management of vascular cognitive impairment. ${ }^{5}$ Evidence for the symptomatic treatment of vascular cognitive impairment is limited. Individuals with poststroke cognitive impairment may benefit from cognitive rehabilitation. A 2016 trial demonstrated that aerobic exercise 3 times a week benefited cognition in individuals with mild vascular cognitive impairment, although the benefit diminished at long-term follow-up. ${ }^{69}$ Trials of acetylcholinesterase inhibitors and memantine have shown mixed results, and these medications are not US Food and Drug Administration (FDA) approved for vascular cognitive impairment. As reviewed earlier, vascular cognitive impairment often co-occurs with Alzheimer pathology, so the use of acetylcholinesterase inhibitors to treat patients with dual pathologies seems logical. Cerebrovascular disease is associated with neuropsychiatric symptoms, including depression. In these cases, treatment with antidepressants such as selective serotonin reuptake inhibitors (SSRIs) can be considered. Some patients with vascular cognitive impairment develop pseudobulbar affect, and an SSRI can potentially mitigate some of the symptoms. Other options include combination dextromethorphan-quinidine, which is FDA approved for the treatment of pseudobulbar affect, although the trials demonstrating its efficacy were done in multiple sclerosis and amyotrophic lateral sclerosis.

\section{Approach to the Patient With Possible Vascular Cognitive Impairment}

As with the evaluation of patients with other causes of cognitive impairment, patients should first be categorized as having normal cognition, mild cognitive impairment, or dementia. In vascular cognitive impairment, the history is important to determine whether a clinical history of stroke or transient ischemic attack is present and assess for traditional cerebrovascular risk factors (eg, smoking, diabetes mellitus, hypertension, hyperlipidemia, atrial fibrillation). The neurologic examination can support a history of cerebrovascular disease with the presence of focal neurologic signs consistent with stroke. Neuroimaging, particularly with MRI, can confirm the presence and extent of cerebrovascular disease, help determine the underlying mechanism, and allow for individualized treatment, including the introduction of secondary prevention strategies. For 
example, in patients with embolic or cortically based infarcts, investigations such as vessel imaging (carotid ultrasound or magnetic resonance/CT angiography), prolonged cardiac monitoring, and echocardiography should be considered to identify and treat the infarct mechanism. In contrast, treatment for individuals with neuroimaging evidence of hypertensive small vessel disease may concentrate on vascular risk factors identified in the clinical evaluation. If cerebral amyloid angiopathy is present on neuroimaging, treatment may focus on minimizing or stopping antithrombotic medications and blood pressure control.

\section{PREVENTIVE STUDIES}

In the Syst-Eur (Systolic Hypertension in Europe) trial, aggressive blood pressure management was associated with a reduced risk of developing dementia compared to placebo. ${ }^{68}$ Similarly, in the PROGRESS (Perindopril Protection Against Recurrent Stroke Study) trial, cognitive decline occurred in 9.1\% of patients on antihypertensive treatment and in $11.0 \%$ on placebo. ${ }^{70}$ The FINGER (Finnish Geriatric Intervention Study to Prevent Cognitive Impairment and Disability) trial investigated a multidomain intervention that included vascular risk factor monitoring and demonstrated a decrease in cognitive decline over 2 years. ${ }^{71} \mathrm{In}$ contrast, several studies have failed to demonstrate reduced risk of dementia or cognitive decline with vascular risk factor treatment. ${ }^{72,73}$ However, the PREDIMED (Prevention With Mediterranean Diet) study, which was a randomized controlled trial of the Mediterranean diet versus a low-fat diet, demonstrated that the Mediterranean diet supplemented with extra virgin olive oil reduced the incidence of cardiovascular events and was associated with improved cognitive function. ${ }^{74}$

\section{CONCLUSION}

Vascular cognitive impairment represents a spectrum of cerebrovascular diseases with different underlying mechanisms. The common co-occurrence of vascular changes in neurodegenerative dementias has reignited interest in treating vascular risk factors to prevent or slow cognitive decline. Prevention in middle age with treatment of vascular risk factors is an important strategy. Recent data support exercise and the Mediterranean diet as emerging treatment strategies, but these lifestyle interventions require additional evidence.

\section{ACKNOWLEDGMENT}

Research reported in this article was supported by the National Institute on Aging/National Institutes of Health (K76AG057015).

\section{REFERENCES}

1 Hachinski VC, Lassen NA, Marshall J. Multi-infarct dementia. A cause of mental deterioration in the elderly. Lancet 1974;2(7874):207-210. doi:10.1016/ s0140-6736(74)91496-2.

2 O’Brien JT, Erkinjuntti T, Reisberg B, et al. Vascular cognitive impairment. Lancet Neurol 2003;2(2): 89-98. doi:10.1016/S1474-4422(03)00305-3.
KEY POINTS

While multi-infarct dementia was once considered synonymous with vascular dementia, it is now recognized that multi-infarct dementia represents a subset of individuals with vascular cognitive impairment.

- Cerebral autosomal dominant arteriopathy with subcortical infarcts and leukoencephalopathy (CADASIL) is a genetic cerebral small vessel disease caused by mutations in the NOTCH3 gene. Cognitive impairment is common in CADASIL, as are migraine headaches and stroke.

T2 hyperintensity involvement of the anterior temporal lobes on MRI may suggest CADASIL as a possible diagnosis.

Cerebral autosomal recessive arteriopathy with subcortical infarcts and leukoencephalopathy (CARASIL) occurs due to mutations in the HTRA1 gene and is associated with alopecia and spondylosis in addition to the cerebral small vessel disease.

Microinfarcts are increasingly recognized as an important contributor to cognitive decline. Recent advances in MRI techniques have allowed a subset to be imaged in vivo.

Treatment of vascular
risk factors in midlife,
aerobic exercise, and a
Mediterranean diet are
promising treatments to
prevent and treat vascular
cognitive impairment but
require further investigation.
research studies. Report of the NINDS-AIREN International Workshop. Neurology 1993;43(2): 250-260. doi:10.1212/WNL.43.2.250. 
4 Gold G, Bouras C, Canuto A, et al. Clinicopathological validation study of four sets of clinical criteria for vascular dementia. Am J Psychiatry 2002;159(1):82-87. doi:10.1176/appi. ajp.159.1.82.

5 Gorelick PB, Scuteri A, Black SE, et al. Vascular contributions to cognitive impairment and dementia: a statement for healthcare professionals from the American Heart Association/American Stroke Association. Stroke 2011;42(9):2672-2713. doi:10.1161/ STR.0b013e3182299496.

6 Sachdev P, Kalaria R, O'Brien J, et al. Diagnostic criteria for vascular cognitive disorders: a VASCOG statement. Alzheimer Dis Assoc Disord 2014;28(3):206-218. doi:10.1097/WAD. 0000000000000034 .

7 American Psychiatric Association. Diagnostic and statistical manual of mental disorders, 5 th ed (DSM-5). Washington, DC: American Psychiatric Association, 2013.

8 Skrobot OA, O'Brien J, Black S, et al. The vascular impairment of cognition classification consensus study. Alzheimers Dement 2017;13(6):624-633. doi:10.1016/j.jalz.2016.10.007.

9 Staekenborg SS, van der Flier WM, van Straaten EC, et al. Neurological signs in relation to type of cerebrovascular disease in vascular dementia. Stroke 2008;39(2):317-322. doi:10.1161/ STROKEAHA.107.493353.

10 Reed BR, Mungas DM, Kramer JH, et al. Profiles of neuropsychological impairment in autopsy-defined Alzheimer's disease and cerebrovascular disease. Brain 2007;130(pt 3): 731-739. doi:10.1093/brain/awl385

11 Mendez MF, Cherrier MM, Perryman KM. Differences between Alzheimer's disease and vascular dementia on information processing measures. Brain Cogn 1997;34(2):301-310. doi:10.1006/brcg.1997.0923.

12 Ruitenberg A, Ott A, van Swieten JC, et al. Incidence of dementia: does gender make a difference? Neurobiol Aging 2001;22(4):575-580. doi:10.1016/S0197-4580(01)00231-7.

13 Knopman DS, Parisi JE, Boeve BF, et al. Vascular dementia in a population-based autopsy study. Arch Neurol 2003;60(4):569-575. doi:10.1001/ archneur.60.4.569.

14 Schneider JA, Arvanitakis Z, Bang W, Bennett DA. Mixed brain pathologies account for most dementia cases in community-dwelling older persons. Neurology 2007;69(24):2197-2204. doi:10.1212/01.wnl.0000271090.28148.24.

15 Schneider JA, Aggarwal NT, Barnes L, et al. The neuropathology of older persons with and without dementia from community versus clinic cohorts. J Alzheimers Dis 2009;18(3):691-701. doi:10.3233/JAD-2009-1227.
16 Hulette C, Nochlin D, McKeel D, et al. Clinical-neuropathologic findings in multi-infarct dementia: a report of six autopsied cases. Neurology 1997;48(3):668-672. doi:10.1212/ WNL.48.3.668

17 Lee JH, Kim SH, Kim GH, et al. Identification of pure subcortical vascular dementia using 11C-Pittsburgh compound B. Neurology 2011;77(1): 18-25. doi:10.1212/WNL.0b013e318221acee.

18 Pendlebury ST, Rothwell PM. Prevalence, incidence, and factors associated with pre-stroke and post-stroke dementia: a systematic review and meta-analysis. Lancet Neurol 2009;8(11):1006-1018. doi:10.1016/ S1474-4422(09)70236-4.

19 Kokmen E, Whisnant JP, O'Fallon WM, et al. Dementia after ischemic stroke: a population-based study in Rochester, Minnesota (1960-1984). Neurology 1996;46(1): 154-159. doi:10.1212/WNL.46.1.154.

20 Jacova C, Pearce LA, Costello R, et al. Cognitive impairment in lacunar strokes: the SPS3 trial. Ann Neurol 2012;72(3):351-362. doi:10.1002/ ana.23733.

21 Vermeer SE, Prins ND, den Heijer T, et al. Silent brain infarcts and the risk of dementia and cognitive decline. N Engl J Med 2003;348(13): 1215-1222. doi:10.1056/NEJMoa022066.

22 Erkinjuntti T, Inzitari D, Pantoni L, et al. Research criteria for subcortical vascular dementia in clinical trials. J Neural Transm Suppl 2000;59: 23-30. doi:10.1007/978-3-7091-6781-6 4.

23 Park JH, Seo SW, Kim C, et al. Pathogenesis of cerebral microbleeds: in vivo imaging of amyloid and subcortical ischemic small vessel disease in 226 individuals with cognitive impairment. Ann Neurol 2013;73(5):584-593. doi:10.1002/ ana.23845.

24 Akoudad S, Wolters FJ, Viswanathan A, et al. Association of cerebral microbleeds with cognitive decline and dementia. JAMA Neurol 2016;73(8):934-943. doi:10.1001/jamaneurol. 2016.1017.

25 Akoudad S, Portegies ML, Koudstaal PJ, et al. Cerebral microbleeds are associated with an increased risk of stroke: the Rotterdam study. Circulation 2015;132(6):509-516. doi:10.1161/ CIRCULATIONAHA.115.016261.

26 Benedictus MR, Prins ND, Goos JD, et al. Microbleeds, mortality, and stroke in Alzheimer disease: the MISTRAL study. JAMA Neurol 2015 72(5):539-545. doi:10.1001/jamaneurol.2015.14.

27 Charidimou A, Boulouis G, Pasi M, et al. $\mathrm{MRI}$-visible perivascular spaces in cerebral amyloid angiopathy and hypertensive arteriopathy. Neurology 2017;88(12):1157-1164. doi:10.1212/WNL.0000000000003746.

28 Banerjee G, Kim HJ, Fox Z, et al. MRI-visible perivascular space location is associated with Alzheimer's disease independently of amyloid burden. Brain 2017;140(4):1107-1116. doi:10.1093/ brain/awx003. 
29 Debette S, Markus HS. The clinical importance of white matter hyperintensities on brain magnetic resonance imaging: systematic review and meta-analysis. BMJ 2010;341:c3666. doi:10.1136/ bmj.c3666

30 Herrmann LL, Le Masurier M, Ebmeier KP. White matter hyperintensities in late life depression: a systematic review. J Neurol Neurosurg Psychiatry 2008;79(6):619-624. doi:10.1136/jnnp.2007.124651

31 Baezner H, Blahak C, Poggesi A, et al. Association of gait and balance disorders with age-related white matter changes: the LADIS study. Neurology 2008;70(12):935-942. doi:10.1212/ 01.wnl.0000305959.46197.e6.

32 Schmidt R, Berghold A, Jokinen $\mathrm{H}$, et al. White matter lesion progression in LADIS: frequency, clinical effects, and sample size calculations. Stroke 2012;43(10):2643-2647. doi:10.1161/ STROKEAHA.112.662593.

33 Maillard P, Seshadri S, Beiser A, et al. Effects of systolic blood pressure on white-matter integrity in young adults in the Framingham Heart Study: a cross-sectional study. Lancet Neurol 2012;11(12):1039-1047. doi:10.1016/S1474-4422(12) 70241-7.

34 Charidimou A, Boulouis G, Haley K, et al. White matter hyperintensity patterns in cerebral amyloid angiopathy and hypertensive arteriopathy. Neurology 2016;86(6):505-511. doi:10.1212/WNL.0000000000002362.

35 McAleese KE, Walker L, Graham S, et al. Parietal white matter lesions in Alzheimer's disease are associated with cortical neurodegenerative pathology, but not with small vessel disease. Acta Neuropathol 2017;134(3):459-473 doi:10.1007/s00401-017-1738-2.

36 van der Flier WM, van Straaten EC, Barkhof F, et al. Small vessel disease and general cognitive function in nondisabled elderly: the LADIS study. Stroke 2005;36(10):2116-2120. doi:10.1161/01 STR.0000179092.59909.42.

37 Benisty S, Gouw AA, Porcher R, et al. Location of lacunar infarcts correlates with cognition in a sample of non-disabled subjects with age-related white-matter changes: the LADIS study. J Neurol Neurosurg Psychiatry 2009;80(5): 478-483. doi:10.1136/jnnp.2008.160440.

38 Jokinen H, Gouw AA, Madureira S, et al. Incident lacunes influence cognitive decline: the LADIS study. Neurology 2011;76(22):1872-1878. doi:10.1212/WNL.0b013e31821d752f.

39 Snowdon DA, Greiner LH, Mortimer JA, et al. Brain infarction and the clinical expression of Alzheimer disease. JAMA 1997;277(10):813-817. doi:10.1001/jama.1997.03540340047031.

40 Arvanitakis Z, Leurgans SE, Wang Z, et al. Cerebral amyloid angiopathy pathology and cognitive domains in older persons. Ann Neurol 2011;69(2): 320-327. doi:10.1002/ana.22112.
41 Pfeifer LA, White LR, Ross GW, et al. Cerebral amyloid angiopathy and cognitive function: the HAAS autopsy study. Neurology 2002;58(11): 1629-1634. doi:10.1212/WNL.58.11.1629.

42 Linn J, Halpin A, Demaerel P, et al. Prevalence of superficial siderosis in patients with cerebral amyloid angiopathy. Neurology 2010;74(17): 1346-1350. doi:10.1212/WNL.0b013e3181dad605

43 van Rooden S, van der Grond J, van den Boom R, et al. Descriptive analysis of the Boston criteria applied to a Dutch-type cerebral amyloid angiopathy population. Stroke 2009;40(9): 3022-3027. doi:10.1161/STROKEAHA.109.554378.

44 DeSimone CV, Graff-Radford J, El-Harasis MA, et al. Cerebral amyloid angiopathy and implications for atrial fibrillation management. Lancet 2017;390(10089):9-11. doi:10.1016/ S0140-6736(17)31326-0.

45 Moulin S, Labreuche J, Bombois S, et al. Dementia risk after spontaneous intracerebral haemorrhage: a prospective cohort study. Lancet Neurol 2016;15(8):820-829. doi:10.1016/ S1474-4422(16)00130-7.

46 Charidimou A, Linn J, Vernooij MW, et al. Cortical superficial siderosis: detection and clinical significance in cerebral amyloid angiopathy and related conditions. Brain 2015;138(pt 5):2126-2139. doi:10.1093/brain/awv162.

47 Gurol ME, Becker JA, Fotiadis P, et al. Florbetapir-PET to diagnose cerebral amyloid angiopathy: a prospective study. Neurology 2016;87(19):2043-2049. doi:10.1212/WNL. 0000000000003197.

48 Carrera E, Bogousslavsky J. The thalamus and behavior: effects of anatomically distinct strokes. Neurology 2006;66(12):1817-1823. doi:10.1212/01.wnl.0000219679.95223.4c.

49 Damasio AR, Graff-Radford NR, Eslinger PJ, Damasio H, Kassell N. Amnesia following basal forebrain lesions. Arch Neurol 1985;42(3):263-271. doi:10.1001/archneur.1985.04060030081013.

50 Graff-Radford J, Williams L, Jones DT, Benarroch EE. Caudate nucleus as a component of networks controlling behavior. Neurology 2017;89(21):2192-2197. doi:10.1212/ WNL.0000000000004680.

51 Dearborn JL, Zhang Y, Qiao Y, et al. Intracranial atherosclerosis and dementia: the Atherosclerosis Risk in Communities (ARIC) study. Neurology 2017;88(16):1556-1563. doi:0.1212/WNL.0000000000003837.

52 Yarchoan $M$, Xie SX, Kling MA, et al. Cerebrovascular atherosclerosis correlates with Alzheimer pathology in neurodegenerative dementias. Brain 2012;135(pt 12):3749-3756. doi:10.1093/brain/aws271.

53 Beach TG, Wilson JR, Sue LI, et al. Circle of Willis atherosclerosis: association with Alzheimer's disease, neuritic plaques and neurofibrillary tangles. Acta Neuropathol 2007;113(1):13-21. doi:10.1007/s00401-006-0136-y 
54 Knopman DS, Mosley TH, Catellier DJ, et al. Fourteen-year longitudinal study of vascular risk factors, APOE genotype, and cognition: the ARIC MRI study. Alzheimers Dement 2009;5(3): 207-214. doi:10.1016/j.jalz.2009.01.027.

55 Graff-Radford J, Madhavan M, Vemuri P, et al. Atrial fibrillation, cognitive impairment, and neuroimaging. Alzheimers Dement 2016;12(4): 391-398. doi:10.1016/j.jalz.2015.08.164.

56 Peters N, Opherk C, Danek A, et al. The pattern of cognitive performance in CADASIL: a monogenic condition leading to subcortical ischemic vascular dementia. Am J Psychiatry 2005;162(11): 2078-2085. doi:10.1176/appi.ajp.162.11.2078.

57 Hara K, Shiga A, Fukutake T, et al. Association of HTRA1 mutations and familial ischemic cerebral small-vessel disease. N Engl J Med 2009;360(17): 1729-1739. doi:10.1056/NEJMoa0801560.

58 Levy E, Carman MD, Fernandez-Madrid IJ, et al. Mutation of the Alzheimer's disease amyloid gene in hereditary cerebral hemorrhage, Dutch type. Science 1990;248(4959):1124-1127. doi:10.1126/science. 2111584 .

59 Levy E, Haltia M, Fernandez-Madrid I, et al. Mutation in gelsolin gene in Finnish hereditary amyloidosis. J Exp Med 1990;172(6):1865-1867. doi:10.1084/jem.172.6.1865.

60 Levy E, Lopez-Otin C, Ghiso J, et al. Stroke in Icelandic patients with hereditary amyloid angiopathy is related to a mutation in the cystatin $\mathrm{C}$ gene, an inhibitor of cysteine proteases. J Exp Med 1989;169(5):1771-1778. doi:10.1084/ jem.169.5.1771.

61 Schneider JA, Boyle PA, Arvanitakis Z, et al. Subcortical infarcts, Alzheimer's disease pathology, and memory function in older persons. Ann Neurol 2007;62(1):59-66. doi:10.1002/ana.21142.

62 Troncoso JC, Zonderman AB, Resnick SM, et al. Effect of infarcts on dementia in the Baltimore longitudinal study of aging. Ann Neurol 2008; 64(2):168-176. doi:10.1002/ana.21413.

63 Barnes DE, Yaffe K. The projected effect of risk factor reduction on Alzheimer's disease prevalence. Lancet Neurol 2011;10(9):819-828. doi:10.1016/S1474-4422(11)70072-2.

64 Sonnen JA, Larson EB, Crane PK, et al. Pathological correlates of dementia in a longitudinal, population-based sample of aging. Ann Neurol 2007;62(4):406-413. doi:10.1002/ ana. 21208 .
65 Arvanitakis Z, Capuano AW, Leurgans SE, et al. The relationship of cerebral vessel pathology to brain microinfarcts. Brain Pathol 2017;27(1):77-85. doi:10.1111/bpa.12365.

66 Raman MR, Preboske GM, Przybelski SA, et al. Antemortem MRI findings associated with microinfarcts at autopsy. Neurology 2014;82(22): 1951-1958. doi:10.1212/WNL.0000000000000471.

67 Graff-Radford J, Raman MR, Rabinstein AA, et al. Association between microinfarcts and blood pressure trajectories. JAMA Neurol 2018;75(2): 212-218. doi:10.1001/jamaneurol.2017.3392.

68 van Veluw SJ, Zwanenburg JJ, Rozemuller AJ, et al. The spectrum of MR detectable cortical microinfarcts: a classification study with 7-tesla postmortem MRI and histopathology. J Cereb Blood Flow Metab 2015;35(5):676-683. doi:10.1038/jcbfm.2014.258.

69 Liu-Ambrose T, Best JR, Davis JC, et al. Aerobic exercise and vascular cognitive impairment: a randomized controlled trial. Neurology 2016; 87(20):2082-2090. doi:10.1212/WNL. 0000000000003332.

70 Tzourio C, Anderson C, Chapman N, et al. Effects of blood pressure lowering with perindopril and indapamide therapy on dementia and cognitive decline in patients with cerebrovascular disease. Arch Intern Med 2003;163(9):1069-1075. doi:10.1001/archinte.163.9.1069.

71 Ngandu T, Lehtisalo J, Solomon A, et al. A 2 year multidomain intervention of diet, exercise, cognitive training, and vascular risk monitoring versus control to prevent cognitive decline in at-risk elderly people (FINGER): a randomised controlled trial. Lancet 2015;385(9984): 2255-2263. doi:10.1016/S0140-6736(15)60461-5.

72 Pearce LA, McClure LA, Anderson DC, et al. Effects of long-term blood pressure lowering and dual antiplatelet treatment on cognitive function in patients with recent lacunar stroke: a secondary analysis from the SPS3 randomised trial. Lancet Neurol 2014;13(12):1177-1185. doi:10.1016/S1474-4422(14)70224-8.

73 Shepherd J, Blauw GJ, Murphy MB, et al. Pravastatin in elderly individuals at risk of vascular disease (PROSPER): a randomised controlled trial. Lancet 2002;360(9346): 1623-1630. doi:10.1016/S0140-6736(02)11600-X.

74 Valls-Pedret C, Sala-Vila A, Serra-Mir M, et al. Mediterranean diet and age-related cognitive decline: a randomized clinical trial. JAMA Intern Med 2015;175(7):1094-1103. doi:10.1001/ jamainternmed.2015.1668. 